\title{
Vibration based Condition Monitoring of a Brake System using Statistical Features with Logit Boost and Simple Logistic Algorithm
}

\author{
Alamelu Mangai. M., Jegadeeshwaran R. , Sugumaran V. \\ School of Mechanical and Building Sciences, VIT University, Chennai Campus, Chennai, 600127, India
}

\begin{abstract}
Brakes are responsible for the stability of the vehicle. Brake failure is one of the key elements where more attention is required. Normally, a brake system failure is not an instantaneous process. It is caused by faults due to reasons like wear, mechanical fade, and oil leak, which started long before the failure progresses. Hence, it is essential to build a model that can recognize the condition from the signal. Condition monitoring is one such supervision approach, which continuously monitors the system and gives characteristics data. These data can be analysed and the condition of the component can be extracted using a machine learning approach. This study focuses on one such machine learning approach using the vibration characteristics of the brake system. The machine learning approach was carried out using feature extraction and feature classification. The statistical information extracted from the vibration signals under various fault conditions were used as features. The features were classified using machine learning algorithms, namely, Simple logistics, Logit boost and Multinominal Regression. Results were compared and discussed. The Logit boost algorithm, which produced $98.91 \%$ classification accuracy, has been suggested as an effective approach for the brake fault diagnosis study.
\end{abstract}

Keywords: fault diagnosis; machine learning; logistics; logit boost; feature classification; multinomial regression

(Submitted on June 30, 2017; Revised on November 22, 2017; Accepted on November 27, 2017)

(C) 2018 Totem Publisher, Inc. All rights reserved.

\section{Introduction}

A brake should be in good condition in order to safely drive a vehicle. It is impossible to control the vehicle without brakes. It is one of the components which should operate even in the harshest conditions. Monitoring such system for faults will reduce the number of accidents and increase the degree of safety. Various reasons for the faults include pad wear, fluid in brakes, mechanical fade, etc. If the fault goes unnoticed, it will result in accidents. Hence, continuous monitoring of brakes will help prevent accidents and will reduce cost by detecting faults at earlier stages.

Condition monitoring is the process of monitoring a system for faults. Fault diagnosis is a systematic approach for detecting faults. Many methods have been reported for detecting faults. Thermal imaging can be used for fault diagnosis of rotary machinery [8]. Model-based fault detection is another method of fault diagnosis [3,7]. Nowadays, vibration analysis has been focused more on fault diagnosis study since the failure symptoms can be identified using the vibration signature. The vibration signal under good conditions and fault conditions can be analyzed through comparison and decisions can be taken [13].

Machine learning is a data mining approach for analysing the vibration signal to make a verdict when intervention is required. Machine learning approach has been utilized for fault diagnosis study [12,17]. In machine learning, the vibration analysis is done through three steps, namely, extraction of features, selection of features and classification of features. Statistical information of a signal was used as features. There are many statistical features [1]. Among them, the contributing features alone were selected for classification using the influence of features [14]. The suggested features were classified using the feature classifier through which the fault categorization accuracy can be predicted. The feature classification process is the process of classifying data into categories. Many classifiers, such as Support Vector Machines [2,6,10], Adapted neuro- 
fuzzy inference system (ANFIS) [6], decision tree and best first tree [9], have been reported for the fault diagnosis of various applications. To enhance the characterization precision a point by point study is required. Hence, an attempt has been made on brake fault diagnosis using Simple logistics, logistics, and multinominal regression algorithms. Figure 1 shows the flow chart of fault diagnosis by using classifiers.

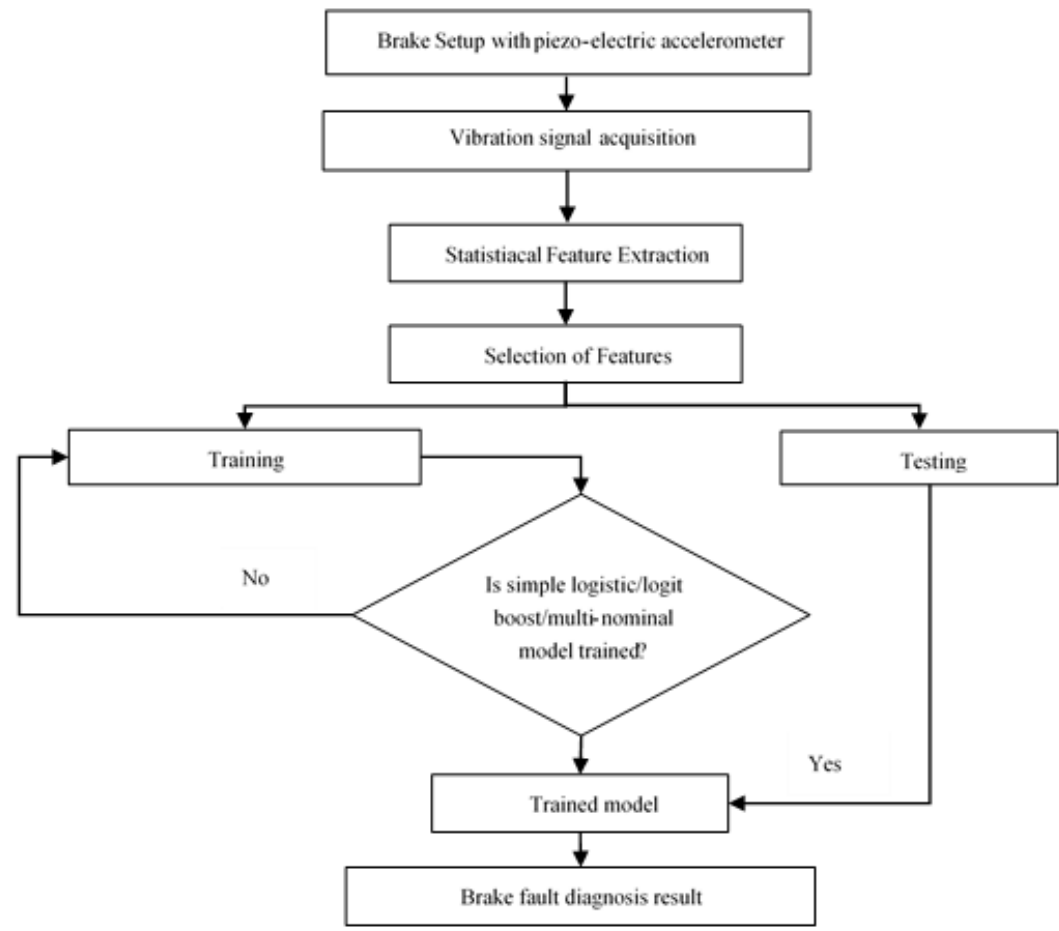

Figure 1. Flow chart for brake fault diagnosis study

Commitments in the present work are as per the following:

- The vibration signals under different fault conditions have been acquired

- Most frequently occurred fault conditions were simulated

- Extracting the relevant statistical information from the acquired vibration signals

- The contributing features alone were selected using the feature selection process

- The suggested feature sets were classified utilizing Simple logistics, Logit Boost and Multinominal Regression

\section{Experimental Setup and Experimental Procedure}

A hydraulic brake system setup was fabricated as an experimental setup (Figure 2). Brake test rig was fabricated from Maruti's hydraulic brake system. The test rig consistis of a drive shaft which includes a disc brake and drum brake. The shaft was driven by an electric Motor (1 HP) using a belt drive. Test rig size is $80 \times 80 \times 20 \mathrm{~cm}$. A piezoelectric uniaxial accelerometer was used to acquire the vibration signals through a data acquisition system (DAQ - Model NI 4432) system. A LabVIEW program was used to store the acquired signals [10].

At first, the brake componenets with good conditions were considered for the initial experiments. The relavant vibration signals were captured using an uni-axial accelerometer from the hydraulic brake system set up under the following settings.

- Drive shaft speed: $60 \mathrm{~km} / \mathrm{Hr}$

- Brake load: $66.67 \mathrm{~N}$

- Sample length: 10000 (arbitrarily chosen)

- Sampling frequency: $24 \mathrm{kHz}$ (as per the Nyquist sampling theorem)

- Sample size: Minimum of 55 samples was taken for each condition of the brake system 


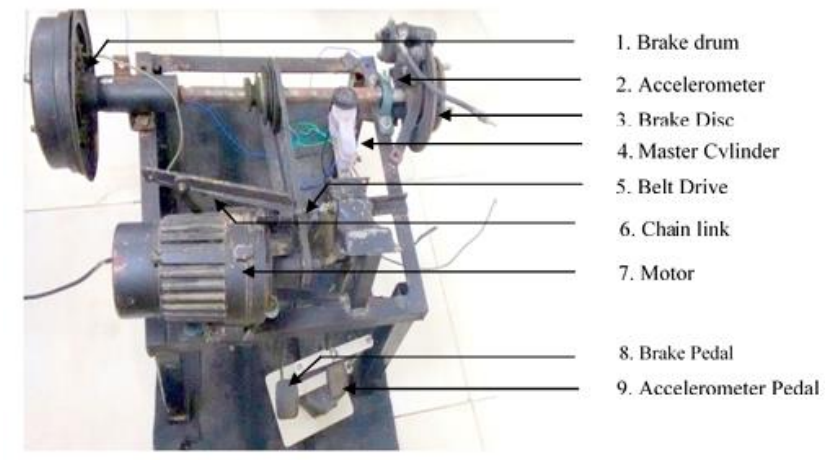

Figure 2. Experimental setup

The most frequently occurring faults were simulated one at a time while other components remained in good condition, and the corresponding vibration signals were acquired [9].
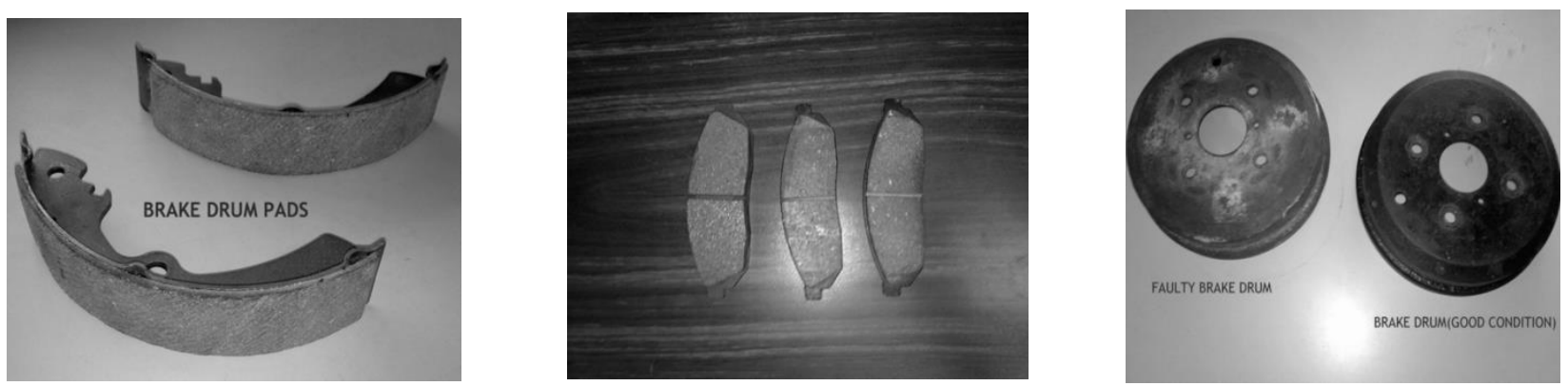

Figure 3. (a) Drum brake pads wear condition; (b) Disc brake pads wear condition; (c) Drum brake mechanical fade

The simulated faults are as follows: Air in the brake fluid (AIR), Oil spill on the disc brake (BOS), Drum brake pad wear (DRPW) (Figure 3 (a)), Inner brake pad wear - uneven (UDPWI), Uneven brake pad wear - inner and outer (UDPWIO), inner brake pad wear - even (DPWI), Even brake pad wear - inner and outer (DPWIO), (Figure 3 (b)), Mechanical fade - drum brake (DRMF) (Figure 3 (c)), Reservoir leak (RL). The vibration signals under each simulated fault conditions were captured. Feature extraction and feature selection were then carried out from the captured signals. Figure 4 shows the schematic of the data acquisition process.

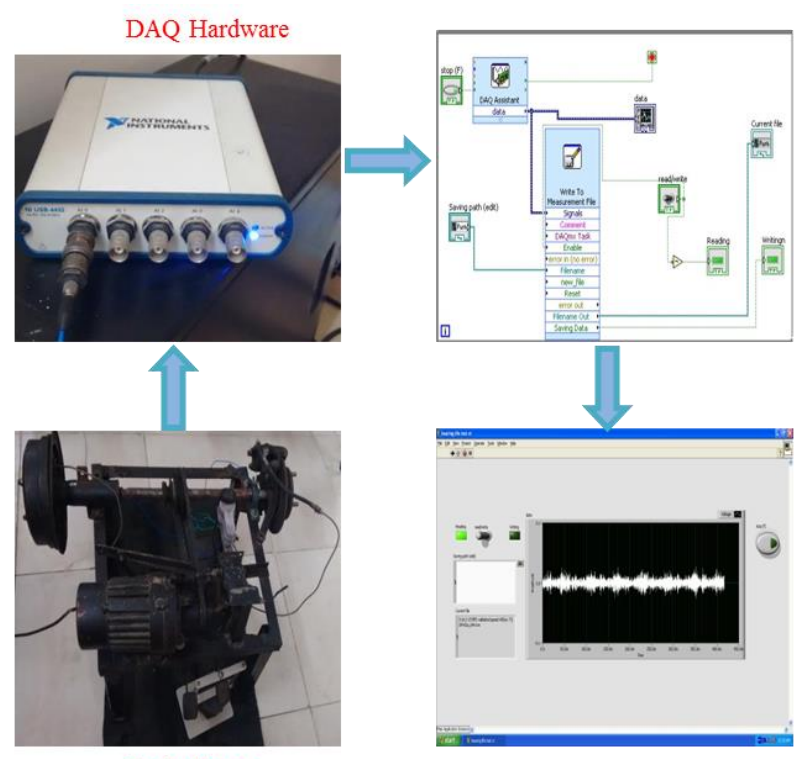

Brake Setup

Figure 4. Schematic of data acquisition process 


\section{Feature Extractions and Selections}

Feature extraction is a process of extracting information from the acquired signals. Feature extraction is a dimensionality reduction approach that simplifies the large set of data by extracting the minimum number of elements that are important. A fairly wide set of statistical parameters, namely standard deviation, mean, mode, count, median, kurtosis, standard error, maximum, sample Variance, minimum and skewness were extracted from vibration signals.

Feature selection is the process of selecting suitable features for building the classification model. It is necessary to remove redundant features from the features set. Irrelevant instances do not provide useful information in any context. In this study, feature selection was carried out using a decision tree. A decision tree algorithm was used to classify the extracted features. The output was obtained as a tree structure called decision tree. Using the top-down approach the contributing features were selected. The feature selection process was verified using the effect of features on the classification accuracy.

\section{Feature Classification}

The selected features were classified using the machine learning algorithms, namely, simple logistic, logit boost and multinomial regression.

\subsection{Simple logistics}

Simple logistics classifier is used for building linear logistic regression models. Logistic regression is used to create some relationship between a categorical response variable and predictor variable. Linear Regression creates a relationship between independent $(X)$ and dependent variable $(Y)$ using a best fit line called a regression line. Logistic regression with independent variable will find the probability that an event may occur (Sumner et al, 2005). Simple linear regression has only one independent variable. The simple logistics can be expressed by an equation

$$
Y=n+m * X+e
$$

where $n$ is intercept, $m$ is slope of the line and $e$ is error term.

The best fit straight line was accomplished by reducing the sum of the squares of the perpendicular deviations from each data point to the line [4].

\subsection{Multi Nominal Regression}

If the power of independent variable is greater than 1 in a regression equation, then, the equation is called as a polynomial regression equation. It constructs a function to set up a relation between weights, outcome, and input.

$$
y=n+m * x^{2}
$$

In this technique, the best fit line is a curve that fits into the data points. From the trained data or outcomes and inputs are known we set up a relationship. It considers the probability of falling into a particular class. Instead of using each variable at a time, it will take into consideration whole classes or categories to find a relationship. Using this relationship, it can predict the outcome if the new data point is given [18].

\subsection{Logit Boost}

Logit boost also known as logistic regression is mainly used for classification. It combines the weak classifiers to get a strong single classifier [12]. Logistic regression with independent variable will find the probability that an event may occur. The value produced is between 0.0 and 1.0. It set some cut point to decide the category. It uses the probability function to predict the category [11].

$$
Y_{i}=e^{U} /\left(1-e^{U}\right)
$$

Where $Y_{i}$ is probability with which $i$ th falls case in a category.

$$
U=A+B_{1} X_{1}+\ldots A_{k} X_{k}
$$


It trains the classifier and gives weight to the features. It reduces the error of classifier at the next iteration. Then, it predicts the value using Bayes rule [5].

$$
Y=\arg \max p k, k=1, \ldots, k
$$

where,

$$
p k=\frac{\exp \left(F_{k}\right)}{\sum_{j=1}^{k} \exp \left(F_{j}\right)}
$$

\section{Results and Discussion}

From the vibration signal, twelve statistical features, namely, standard error, count, sample variance, mean, kurtosis, median, skewness, standard deviation, minimum, maximum, range, sum were extracted from the vibration signals. Using the effect of a number of features study, the feature selection was done.

\subsection{Effect of Number of Features}

Twelve statistical features were classified using the decision tree algorithm. The decision tree was obtained as an output. It shows the contributing features in the form of the top-down approach. Effect of number of features is shown in Table 1. Using the decision tree, the order of features was decided according to the effect of each feature on classification. For each set of features, the classification accuracy was noted down. Maximum accuracy is given by Logit boost with seven features. From the logit boost, the seven features, namely, mean, kurtosis, standard Error, median, sample variance, standard deviation, and skewness were selected. Fall of accuracy due to as number accuracy increases the complexity increases and it confuses the classifier. Using the decision tree, the order of features was decided based on the effect of each feature on the classification accuracy. For each set of features, the classification accuracy was noted down. Maximum accuracy is given by Logit boost with seven features.

From the logit boost, the following seven features were selected: Mean, Standard Error, Median, Standard Deviation, Sample Variance, Kurtosis, and Skewness. Fall of accuracy due to as number accuracy increases the complexity increases and it confuses the classifier.

Table 1. Effect of Number of Features

\begin{tabular}{|c|c|c|c|c|}
\hline \multirow{2}{*}{ S. No } & \multirow{2}{*}{ Number of Features } & \multicolumn{3}{|c|}{ Classifier accuracy (\%) } \\
\cline { 3 - 5 } & 1 & Simple logistics & Multi nominal regression & Logit Boost \\
\hline 1 & 2 & 47.45 & 49.82 & 49.55 \\
\hline 2 & 3 & 86.91 & 86.73 & 90.55 \\
\hline 3 & 4 & 87.82 & 87.45 & 90.55 \\
\hline 4 & 5 & 92.18 & 95.45 & 97.27 \\
\hline 5 & 6 & $\mathbf{9 3 . 8 2}$ & 96.91 & 97.82 \\
\hline 6 & 7 & 93.09 & 97.45 & 97.82 \\
\hline 7 & 8 & 91.45 & 97.45 & 98.36 \\
\hline 8 & 9 & 91.45 & 96.91 & 98.36 \\
\hline 9 & 10 & 91.45 & 97.27 & 98.36 \\
\hline 10 & 11 & 89.27 & 97.64 & 98.36 \\
\hline 11 & 12 & 89.27 & 97.64 & 98.36 \\
\hline 12 & & 89.27 & 97.64 & \\
\hline
\end{tabular}

\subsection{Feature Classification using Simple Logistics}

Referring to Table 1, the simple logistic algorithm produced maximum classification accuracy with five features. The top five features alone were separated from the all feature set and were classified using the simple logistics algorithm. The obtained results were shown in a confusion matrix (Table 2).

In the confusion matrix, the diagonal element shows the data points that were correctly classified and the nondiagonal elements that were misclassified data points. The first element in the first row in the confusion matrix represents the number of data sets corresponding to "GOOD" condition. In the confusion matrix, the first column (except diagonal element) represents how many data sets were wrongly categorized as the other fault condition. The first element in the first column signifies how many data points were correctly classified as "GOOD" condition. Among the 55 data sets belonging to GOOD 
condition, all data points were categorized correctly as "GOOD" condition. Hence there was no mis-classification. Similarly, the second element in the second row in the confusion matrix represents "AIR" condition. The second element in the second row column refers to how many data sets belong to "AIR" condition, and how many were classified correctly as "AIR" condition. The non-diagonal elements represent how many data sets belonging to the "AIR" condition that were wrongy classified as other fault conditions. In this fashion, among the 55 data sets, 54 data points were categorized correctly as "AIR", and one data point was wrongly classified as "DRPW" condition. In this fashion, the classification accuracies were found and the following results were obtained. In this case, the classification accuracy was obtained as $93.82 \%$.

Total Number of data points Correctly Classified data points Incorrectly Classified data points Root mean squared error
550

$516 \quad 93.82 \%$

$34 \quad 6.18 \%$

0.1175

Table 2. Confusion matrix for Simple logistics algorithm

\begin{tabular}{|c|c|c|c|c|c|c|c|c|c|c|}
\hline Category & GOOD & AIR & BOS & DPWI & DPWIO & UDPWI & UDPWIO & DRMF & DRPW & RL \\
\hline GOOD & 55 & 0 & 0 & 0 & 0 & 0 & 0 & 0 & 0 & 0 \\
\hline AIR & 0 & 54 & 0 & 0 & 0 & 0 & 0 & 0 & 1 & 0 \\
\hline BOS & 0 & 0 & 53 & 0 & 0 & 2 & 0 & 0 & 0 & 0 \\
\hline DPWI & 0 & 1 & 2 & 49 & 0 & 3 & 0 & 0 & 0 & 0 \\
\hline DPWIO & 4 & 2 & 0 & 0 & 49 & 0 & 0 & 0 & 0 & 0 \\
\hline UDPWI & 0 & 0 & 0 & 1 & 0 & 54 & 0 & 0 & 0 & 0 \\
\hline UDPWIO & 0 & 0 & 6 & 1 & 0 & 0 & 48 & 0 & 0 & 0 \\
\hline DRMF & 0 & 0 & 0 & 0 & 0 & 2 & 0 & 53 & 0 & 2 \\
\hline DRPW & 0 & 5 & 0 & 0 & 0 & 0 & 0 & 3 & 47 & 0 \\
\hline RL & 0 & 0 & 0 & 0 & 0 & 0 & 0 & 1 & 0 & 54 \\
\hline
\end{tabular}

\subsection{Feature Classification using Multi Nominal Regression}

Referring to Table 1, the multinominal regression algorithm produced maximum classification accuracy at ten features. The top ten features, namely, standard error, skewness, sample variance, kurtosis, minimum, mean, median, maximum, range and standard deviation, were selected and were classified using the multinominal regression algorithm. The result was obtained as a confusion matrix and is shown in Table 3. The overall classification accuracy of multi nominal regression was $97.64 \%$.

Total Number of data points Correctly Classified data points Incorrectly Classified data points Root mean squared error
550

537

13

0.0687
$97.64 \%$

$2.36 \%$

Table 3. Confusion matrix for Multinominal logistic regression algorithm

\begin{tabular}{|c|c|c|c|c|c|c|c|c|c|c|}
\hline Category & GOOD & AIR & BOS & DPWI & DPWIO & UDPWI & UDPWIO & DRMF & DRPW & RL \\
\hline GOOD & 55 & 0 & 0 & 0 & 0 & 0 & 0 & 0 & 0 & 0 \\
\hline AIR & 0 & 54 & 0 & 1 & 0 & 0 & 0 & 0 & 0 & 0 \\
\hline BOS & 0 & 0 & 54 & 0 & 0 & 0 & 1 & 0 & 0 & 0 \\
\hline DPWI & 1 & 0 & 0 & 54 & 0 & 0 & 0 & 0 & 0 & 0 \\
\hline DPWIO & 0 & 0 & 0 & 1 & 52 & 1 & 0 & 0 & 0 & 0 \\
\hline UDPWI & 0 & 0 & 0 & 1 & 0 & 54 & 0 & 0 & 0 & 0 \\
\hline UDPWIO & 0 & 0 & 1 & 0 & 0 & 0 & 53 & 0 & 0 & 1 \\
\hline DRMF & 0 & 0 & 0 & 0 & 0 & 0 & 0 & 54 & 0 & 1 \\
\hline DRPW & 0 & 0 & 0 & 1 & 0 & 0 & 0 & 0 & 53 & 1 \\
\hline RL & 0 & 0 & 0 & 0 & 0 & 0 & 0 & 1 & 0 & 54 \\
\hline
\end{tabular}

\subsection{Feature Classification using Logit boost algorithm}

Referring to Table 1, the Logit boost (Logistic regression) algorithm produced maximum classification accuracy at seven features. The top seven features, namely, median, standard error, kurtosis, sample variance, skewness, minimum and mean were selected. The logit boost algorithm was used for classification. Table 4 shows the results obtained from the algorithm. The classification accuracy of Logit boost algorithm was $98.91 \%$. Comparatively, the logit boost produced better classification accuracy than the other two classifiers, namely, simple logistics and multinominal logistics algorithm. The detailed accuracy by class of the logit boost is shown in Table 5. The following summarized results were obtained through logit boost algorithm. 
Total Number of data points Correctly Classified data points Incorrectly Classified data points Root mean squared error
550

544

6

0.0455
$98.91 \%$

$1.09 \%$

Table 4. Confusion matrix for Logit boost Algorithm

\begin{tabular}{|c|c|c|c|c|c|c|c|c|c|c|}
\hline Category & GOOD & AIR & BOS & DPWI & DPWIO & UDPWI & UDPWIO & DRMF & DRPW & RL \\
\hline GOOD & 55 & 0 & 0 & 0 & 0 & 0 & 0 & 0 & 0 & 0 \\
\hline AIR & 0 & 54 & 0 & 1 & 0 & 0 & 0 & 0 & 0 \\
\hline BOS & 0 & 0 & 55 & 0 & 0 & 0 & 0 & 0 & 0 \\
\hline DPWI & 0 & 0 & 0 & 55 & 0 & 0 & 0 & 0 & 0 & 0 \\
\hline DPWIO & 0 & 0 & 0 & 0 & 55 & 0 & 0 & 0 & 0 & 0 \\
\hline UDPWI & 0 & 0 & 0 & 0 & 0 & 55 & 0 & 0 & 0 & 0 \\
\hline UDPWIO & 0 & 0 & 0 & 0 & 0 & 0 & 55 & 0 & 0 & 0 \\
\hline DRMF & 0 & 0 & 0 & 1 & 0 & 0 & 1 & 52 & 0 & 1 \\
\hline DRPW & 0 & 0 & 0 & 0 & 0 & 0 & 0 & 1 & 54 & 0 \\
\hline RL & 0 & 0 & 0 & 0 & 0 & 0 & 0 & 1 & 0 \\
\hline
\end{tabular}

Table 5: Detailed Accuracy by Class - Logit boost

\begin{tabular}{|c|c|c|c|c|c|c|}
\hline TP Rate & FP Rate & Precision & Recall & F-Measure & ROC Area & Class \\
\hline 1 & 0 & 1 & 1 & 1 & 1 & GOOD \\
\hline 0.982 & 0 & 1 & 0.982 & 0.991 & 1 & AIR \\
\hline 1 & 0 & 1 & 1 & 1 & 1 & BOS \\
\hline 1 & 0.004 & 0.965 & 1 & 0.982 & 1 & DPWI \\
\hline 1 & 0 & 1 & 1 & 1 & 1 & DPWIO \\
\hline 1 & 0 & 1 & 1 & 1 & 1 & UDPWI \\
\hline 1 & 0.002 & 0.982 & 1 & 0.991 & 1 & UDPWIO \\
\hline 0.945 & 0.004 & 0.963 & 0.945 & 0.954 & 0.998 & DRMF \\
\hline 0.982 & 0 & 1 & 0.982 & 0.991 & 1 & DRPW \\
\hline 0.982 & 0.002 & 0.982 & 0.982 & 0.982 & 1 & RL \\
\hline 0.989 & 0.001 & 0.989 & 0.989 & 0.989 & 1 & WT. AVG \\
\hline
\end{tabular}

Referring to Table 5, the detailed accuracy by individual class can be studied. In pattern recognition, precision is the fraction of retrieved instances that are relevant, while recall is the fraction of relevant instances that are retrieved. True Positive (TP) rate measures the proportion of positives that are correctly identified as true, and False Positive (FP) rate measures the proportion of negatives that are correctly identified as a false class. For an ideal classifier, TP rate should be 1 and the FP rate should be 0. For GOOD condition, there is no misclassification; hence, the TP rate is 1 and FP rate is zero. For RL condition, one data point has been misclassified as other fault condition. Hence, the true positive rate is $0.989(54 / 55)$ and the FP rate is $0.001(1 / 55)$. Overall, the classification accuracy is maximum with the logit boost algorithm. Table 6 shows the comparative results of all other classifiers.

Table 6. Comparative results
\begin{tabular}{|l|c|}
\hline \multicolumn{1}{|c|}{ Name of the classifier } & Classification accuracy (\%) \\
\hline Simple logistics algorithm & 93.82 \\
\hline Multi-nominal regression algorithm & 97.64 \\
\hline Logit Boost Algorithm & 98.91 \\
\hline
\end{tabular}

\section{Conclusions}

In this paper, the simple logistics, multinominal regression and logit boost algorithms were used as classifiers to classify the vibration data from the brake test rig. The frequently arising faults were considered. The vibration signal was acquired under each simulated fault condition using an accelerometer. From the vibration signals, twelve sets of statistical features were extracted. The dominant features alone were selected for classification. The suggested features were classified using simple logistics, multinominal regression and logit boost algorithm. Results were compared and it is observed that the logit boost with seven features can give the maximum accuracy of $98.91 \%$. Hence, the logit boost classifier can be used for the brake fault diagnosis study for reducing accidents and improving safety on the road.

\section{References}

1. A. Bahri, V. Sugumaran, R. Jegadeeshwaran and S. B. Devasenapathi, "Misfire Detection in Spark-Ignition Engine using Statistical Learning Theory," International Journal of Performability Engineering, vol. 12, no. 1, pp. 79-88.

2. L. Batista, B. Badri, R. Sabourin and M. Thomas, "A classifier fusion system for bearing fault diagnosis", Expert Systems with 
Applications, vol.40, no. 1, pp.6788-6797, 2013.

3. M. Borner, H. Straky, T. Weispfenning and R. Isermann. "Model-based fault detection of vehicle suspension and hydraulic brake systems", Mechatronics, Vol.12, No. 1, pp. 999-1010, 2002.

4. J. Friedman, T. Hastie and R. Tibshirani, "Additive logistic regression: A statistical view of boosting," The Annuals of statistics 2000, vol. 28, no.2, pp.337-407, 2000.

5. Y-L He, R. Wang, S. Kwong and X-Z Wang, "Bayesian classifiers based on probability density estimation and their applications to simultaneous fault diagnosis," Information Sciences, vol. 259, no. 1, pp. 252-268, 2014.

6. K. Salahshoor, K. Mojtaba and M. S. Khoshro, "Fault detection and diagnosis of an industrial steam turbine using fusion of SVM (support vector machine) and ANFIS (adaptive neuro-fuzzy inference system) classifiers", Energy, vol.35, pp. 5472-5482, 2010.

7. A. K. Jalan and A. R. Mohanty, "Model based fault diagnosis in rotating machinery," International Journal of Performability Engineering, vol. 7, no. 6, pp. 515-523, 2011.

8. O. Janssens, R. Schulz, V. Slavkovikj, K. Stockman, M. Loccufier, R. van de Walle and S. Van Hoecke. "Thermal image based fault diagnosis for rotating machinery", Infrared Physics \& Technology, vol. 73, pp. 78-87, 2015.

9. R. Jegadeeshwaran, and V. Sugumaran, "Comparative study of decision tree classifier and best first tree classifier for fault diagnosis of automobile hydraulic brake system using statistical features", Measurement, vol. 46, no. 9, pp.3247-3260, 2013.

10. R. Jegadeeshwaran, and V. Sugumaran, "Fault diagnosis of automobile hydraulic brake system using statistical features and support vector machines", Mechanical systems and signal processing, vol. 52-53, no. 1, pp. 436-446, 2015.

11. J. Padmavathi, "Logistic regression in feature selection in data mining", International Journal of Scientific \& Engineering Research, vol. 3, no. 8, 2012.

12. H-W Peng and P-J Chiang, "Control of Mechatronics Systems Ball Bearing Fault Diagnosis Using Machine Learning Techniques", Proceedings of 2011 8th Asian Control Conference (ASCC) Kaohsiung, Taiwan, 2011.

13. N. R. Sakthive, V. Sugumaran, and S. Babudevasenapati, "Vibration based fault diagnosis of monoblock centrifugal pump using decision tree", Expert Systems with Applications, vol. 37, no. 6, pp. 4040-4049, 2010

14. V. Sugumaran, and K. I. Ramachandran, "Effect of number of features on classification of roller bearing faults using SVM and PSVM", Expert Systems with Applications, vol. 38, no. 4, pp. 4088-4096, 2011.

15. M. Sumner, E. Frank, M. Hall, "Speeding up Logistic Model Tree Induction," In: 9th European Conference on Principles and Practice of Knowledge Discovery in Databases, 675-683, 2005.

16. P. Sun, M. D. Reid and J. Zhou, "AOSO-Logit Boost: Adaptive One-Vs-One Logit Boost for Multi-Class Problem," 2012.

17. C-C Wang, C-W Lee and C-S Ouyang. "A Machine-Learning-Based Fault Diagnosis Approach for Intelligent Condition Monitoring", Ninth International Conference on Machine Learning and Cybernetics, Qingdao, 2014

18. K. Watanabe and T. Kurita, "Locality Preserving Multi-nominal Logistic Regression," 2008. 